\begin{tabular}{l}
\hline Alimentary Pharmacology \\
\hline \& Therapeutics \\
\hline
\end{tabular}

\title{
Review article: Faecal transplantation therapy for gastrointestinal disease.
}

\begin{tabular}{|c|c|}
\hline Journal: & Alimentary Pharmacology \& Therapeutics \\
\hline Manuscript ID: & APT-0357-2011.R2 \\
\hline Wiley - Manuscript type: & Review Article \\
\hline $\begin{array}{r}\text { Date Submitted by the } \\
\text { Author: }\end{array}$ & 23-May-2011 \\
\hline Complete List of Authors: & $\begin{array}{l}\text { Landy, Jonathan; St Mark's Hospital, IBD Unit; Imperial College } \\
\text { University, APRG } \\
\text { Omar, Hafid; Imperial College University, APRG } \\
\text { McLaughlin, simon; Royal Bournemouth Hospital, Gastroenterology } \\
\text { Walker, Alan; Wellcome Trust Sanger Institute, Wellcome Trust } \\
\text { Genome Campus } \\
\text { Ciclitira, Paul; The Rayne Institute, St Thomas' Hospital } \\
\text { Nicholls, John; Imperial College, Department of Biosurgery and } \\
\text { Surgical Technology } \\
\text { Clark, Sue; St. Mark's Hospital, Surgery } \\
\text { Hart, Ailsa; St Mark's Hospital, IBD Unit; Imperial College } \\
\text { University, APRG }\end{array}$ \\
\hline Keywords: & $\begin{array}{l}\text { Enteric infections < Disease-based, Alternative medicine < Topics, } \\
\text { Microbiology < Topics, Probiotics/prebiotics < Topics, Inflammatory } \\
\text { bowel disease }<\text { Disease-based }\end{array}$ \\
\hline
\end{tabular}




\section{Review article: Faecal transplantation therapy for gastrointestinal disease.}

(Short Title) Review article: Faecal transplantation

\section{Landy J 1,2, Al-Hassi HO 2, McLaughlin SD 3, Walker AW 4, Ciclitira PJ 5, Nicholls RJ 6, Clark SK 7, Hart AL 1,2,}

Keywords: Clostridium difficile, Dysbiosis, Microbiota, Faecal transplantation, Bacteriotherapy

1. IBD Unit, St Mark's Hospital, Harrow, London, UK

2. Antigen Presentation Research Group, St Mark's Campus, Imperial College, London, UK

3. Department of Gastroenterology, Royal Bournemouth Hospital, Bournemouth, UK

4. Wellcome Trust Sanger Institute, Wellcome Trust Genome Campus, Hinxton, Cambridge, UK

5. Department of Gastroenterology, The Rayne Institute, St Thomas' Hospital, London, UK

6. Department of Biosurgery and Surgical Technology, Imperial College, London, UK

7. Department of Surgery, St Mark's Hospital, Harrow, London, UK

Corresponding Author:

Dr A Hart

IBD Unit, $4^{\text {th }}$ Floor, St Mark's Hospital, Harrow, London, HA1 3UJ

Tel- 02082354000

Fax- 02082354093

Email: ailsa.hart@nwlh.nhs.uk 


\begin{abstract}
Background: Evidence is emerging regarding the relationship between a dysbiosis of the human gut microbiota and a number of gastrointestinal diseases as well as diseases beyond the gut. Probiotics have been investigated in many gastrointestinal disease states, with variable and modest outcomes. Faecal transplantation is an alternative approach to manipulate the gut microbiota. Aims: To review the use of faecal transplantation therapy for the management of gastrointestinal disorders.

Methods: Available articles on faecal transplantation in the management of gastrointestinal disorders, were identified through a Pubmed search and bibliographies of review articles on the subject were collated. Results: 239 patients who had undergone faecal transplantation were reported. Seventeen of twenty two studies of faecal transplantation were in fulminant or refractory Clostridium difficile. Studies of faecal transplantation are heterogeneous regarding the patients, donors, screening, methods of administration, and definition of response. Faecal transplantation for Clostridium difficile has been demonstrated to be effective in 145/166 (87\%) patients. Small numbers of patients are reported to have undergone successful faecal transplantation for irritable bowel syndrome and inflammatory bowel disease. Conclusions: Faecal transplantation has been reported with good outcomes for
\end{abstract}




\author{
fulminant and refractory Clostridium difficile. No adverse \\ effects of faecal transplantation have been reported. However, \\ there are no level 1 data of faecal transplantation and reports to \\ date may suffer from reporting bias of positive outcomes and \\ under-reporting of adverse effects. This therapy holds great \\ promise where a dysbiosis of the gut microbiota is responsible \\ for disease and further studies are necessary to explore this \\ potential.
}

\title{
Background
}

The possibility of modifying the gut microbiota to replace harmful bacteria with more favourable microbes has been widely explored since Metchnikoff's observations in 1907 of the potential health benefits of the "Bulgarian bacillus" (1). With the application of molecular techniques to the study of gut microbiology, mounting evidence is emerging regarding the relationship between a dysbiosis of the human gut microbiota and a number of gastrointestinal diseases as well as diseases beyond the gut including diabetes and metabolic syndrome $(2,3,4,5)$.

In vitro studies have demonstrated a positive effect of probiotic bacteria on gut inflammation by modulating gut immune cells $(6,7)$. Probiotics have been extensively investigated in many 
gastrointestinal disease states where an abnormal microbiota is considered pathogenic $(8,9,10)$. The outcomes of these studies have however been variable and modest (10). One confounding factor of the probiotic approach is the comparatively low number and diversity of bacterial species available in a typical commercial probiotic preparation in comparison with the gut microbiota. Furthermore, probiotic bacterial strains may not be able to compete effectively against the complex interactions of an established and adapted indigenous gut microbial community.

An alternative approach is transplantation of the gut microbiota. This is a concept that has been described in ruminants for some time (11). Its use as therapy in humans was first reported by Eiseman et al. in 1958 in the treatment of fulminant pseudomembranous enterocolitis (12). Over the subsequent decades, there have been a small number of case reports and case series of faecal transplantation for Clostridium difficile (13-29) and also constipation (16,30,31), irritable bowel syndrome $(16,30)$ and inflammatory bowel diseases $(16,30,32,33)$. In recent years there has been a resurgence of interest in this procedure and its potential to modify the gut microbiota.

Reports of the procedure have originated from Canada and the United States, Australia and Northern Europe, but the methods of faecal transplantation, screening of donors and patient 
groups treated with this therapy have varied greatly. In this article, we review the use of faecal therapy since the 1958 report of Eiseman et al. Available articles on the use of faecal transplantation in the management of human gastrointestinal disorders, which were identified through a Pubmed search (15.1.11) and bibliographies of review articles on the subject were collated. Articles including patients that were previously described or articles that were not available in English were not reviewed. The included publications encompassed different gastrointestinal pathologies, varying methods of treatment, screening and duration of follow up. Twenty two reports of faecal transplantation meeting the inclusion criteria, were identified. Ten of these were published since 2005, demonstrating the recently renewed interest in this area. In total, there are 239 patients who have undergone faecal transplantation reported.

\section{Patient Details}

The majority of patients undergoing faecal transplantation were treated for Clostridium difficile after standard treatments had failed. Borody et al. in 1989 (16) reported 55 patients treated for constipation, diarrhoea, abdominal pain, ulcerative colitis or Crohn's disease. This report did not specify the numbers of patients with each condition, although out of five cases described in more detail, two patients had irritable bowel 
syndrome, one ulcerative colitis, one Crohn's disease and one Clostridium difficile diarrhoea. Andrews et al. (31) described faecal enema treatment for two patients with constipation and in the recent paper from Grehan et al. (32), nine patients had a diagnosis of constipation or diarrhoea predominant IBS and one patient had Crohn's disease. One patient in the series from Aas et al. (20) had Clostridium difficile diarrhoea on a background of Crohn's colitis. Seven other patients with ulcerative colitis are reported to have undergone faecal transplantation $(32,33)$.

Faecal transplantation has been described in patients as young as two years old (24) to patients over 90 years of age (23). Several reports include patients with serious co-morbidities. Three of the four patients reported by Eiseman et al. (12) were in a critical condition requiring the use of vasopressors. In the patients reported by Bowden et al. (13), eight had a previously treated carcinoma, two chronic renal failure and two an aortic aneurysm. In the study by Aas et al. (20) five patients undergoing faecal transplantation were hospitalised and of those treated as outpatients, three were nursing home residents. MacConnachie et al. (22) described faecal transplantation in eighteen patients, eleven of whom were hospitalised with significant co-morbidity and a high proportion having hypoalbuminaemia, leucocytosis and renal dysfunction before faecal transplantation. The patient in the report of You et al. 
(21) was treated in an intensive care unit with vasopressors and continuous veno-venous haemofiltration.

\section{Donor Screening}

The potential risk of transmission of viral, bacterial or parasitic infection during the course of faecal transplantation is a concern. No guidelines currently exist regarding screening before faecal transplantation. A number of studies have proposed screening procedures $(20,24)$. In a recent review of faecal tranplantation for recurrent Clostridium difficile (34) Bakken suggests a screening process based on previous studies. However, without established guidelines or data from randomised controlled trials, ethical approval for the procedure has to date depended on physician discretion with patient and donor consent, local hospitals' or authorities' approval or occurred within the framework of ethically approved research studies.

Screening methods of stool donors are not always detailed. In the majority of reports a spouse or partner, close relative, or household member of the patient is preferred as the stool donor. However, in a number of reports, donors who are unrelated healthy individuals have been used $(13,18,33)$. Earlier cases did not employ rigorous screening protocols, whereas more 
recently, increased screening of donors' medical histories, blood and stool tests have been implemented.

Donors have been screened for a history of gastrointestinal illness, cancer or polyps, hospitalisation within the three previous months (25) and between 6 weeks (33) to 6 months (20) without the use of antibiotics. Screening blood tests have included full blood count and liver function tests (31) as well as screening of viral pathogens including HIV 1+2 (17-20,2227,29,32), HTLV I/II (25) hepatitis A, B and C (18-20,22-25,29,33), CMV, EBV (18,33) and also for Treponema pallidum (20,2224,32) and Helicobacter pylori antibody (25).

Donor faecal specimens have been screened for Salmonella spp., Shigella spp., Campylobacter spp., Staphylococcus aureus, Aeromonas hydrophila, Yersinia spp., Vibrio parahaemolyticus, Vibrio cholerae, Candida albicans, Escherichia coli $\mathrm{O} 157$ and Clostridium difficile toxins A and B $(17,18,20,22-29,33)$. Stool microscopy has been screened for protozoa (trophozoites and cysts), helminths and ova including Entamoeba histolytica, Giardia lamblia, Microspora spp. (20,22-25,27,33), Cryptosporidium spp. (25), Dientamoeba fragilis, Blastocystis hominis, Ascaris lumbricoides, trematodes and tape worms (20,22-25,27,33).

(Table 1). 


\section{Route of Administration}

The initial report of Eiseman et al. described administration of faecal enemas (12), which has been replicated in other studies (13-15,17,18,21,25,30-33). Others subsequently have used instillation via a colonoscope to the right colon $(19,26-30)$ or instillation of donor faeces via nasogastric tube $(20,22-24)$ or duodenal (29) or nasojejunal intubation $(13,30)$. The study of Grehan et al. employed a combination of colonoscopic instillation followed by enemas or nasojejunal tube (30). The majority of studies entailed a single administration of donor faeces. Some studies used repeated infusions over 2 to 15 days (12-15,17,31,33). In the study by Garborg et al. (29), six patients underwent a second infusion of donor faeces having not responded to the initial transplantation.

\section{Patient preparation}

Preparation of the patient prior to faecal transplantation has varied depending on the method of administration of the donor stool. Studies in which donor stool is instilled at colonoscopy or via rectal enemas include patient preparation with bowel lavage treatments. Bennet and Brinkmann describe a bowel sterilisation procedure (32) prior to transplantation of donor 
stool. Persky and Brandt described the use of prior bowel lavage with polyethylene glycol (19). The series of Borody et al. in six patients with refractory ulcerative colitis, gave seven to ten days of treatment with vancomycin, metronidazole and rifampicin prior to bowel lavage (33). This protocol was repeated in the study by Grehan et al. (32). Two recent studies stopped treatment with metronidazole or vancomycin 24-48 hours prior to faecal transplantation $(27,29)$. The study by Silverman et al., included prior treatment with Saccharomyces boulardii which was continued up to 60 days after the procedure (25). Patients treated at one centre in the study by Rholke et al. (26) were treated with loperamide immediately following the procedure and again 6 hours later in order maximise contact time of the donor faeces with the colonic mucosa.

Studies of faecal transplantation administered into the upper gastrointestinal tract, do not report the use of prior bowel lavage. The method described by Aas et al. in 2003 and followed by those of MacConnachie, Rubin and Russell et al., includes pre-treatment with more than four days of vancomycin and $20 \mathrm{mg}$ of omeprazole the evening before and the morning of the faecal transplantation procedure (20,22-24).

\section{Preparation of donor stool}


The interval between obtaining donor stool and its administration to the patient has varied between studies, from 24 hours before, 6 hours before (20,22-24) or immediately. One study homogenised donor stool in pasteurised cow's milk and filtered the solution which was then stored at $-20^{\circ} \mathrm{C}$ and thawed in water at $37^{\circ} \mathrm{C} 30-60$ minutes prior to administration as an enema (18). Some studies have described the homogenisation of the stool and filtering to remove debris. The use of between 10 to $200 \mathrm{~g}$ of stool, diluted in $20-500 \mathrm{ml}$ sterile saline, has been reported depending on the method of administration. Studies using an upper gastrointestinal protocol for faecal transplantation instilled between 30 and $50 \mathrm{~g}$ of stool homogenised with 50-250ml sterile saline. (Table 2).

\section{Outcomes}

In many reports of faecal transplantation response is not clearly defined. Resolution of symptoms is most commonly stated. Some papers include absence of Clostridium difficile toxin. In the 1989 paper by Borody et al., the indication for faecal transplantation in 50 of 55 patients treated was not stated. In this study however, 20 patients were cured, 26 responded and 9 patients did not respond to faecal transplantation (16). In the paper by Grehan et al., outcomes were not stated (30).

Excluding these studies, faecal transplantation for Clostridium difficile has been demonstrated to be effective in 145/166 
(87\%) patients. Time to response is often not stated, although "immediate", "prompt" or "rapid" response is often reported. Where time to response is stated, this has been recorded to occur within 24 hours to twelve days $(13,18,24,18,29,33)$.

Response appears durable with follow up of patients up to 8 years following faecal transplantation (27).

In the initial report of Eiseman et al. three of the four patients were described as terminally or critically ill. All of these had cessation of diarrhoea and were completely asymptomatic between 24 hours and ten days following faecal transplantation. The report of Bowden et al. describes response as a reduction in frequency of bowel motions, absence of fever, normalisation of leucocyte counts and increased general well being. Tvede and Rask-Madsen describe normalisation of bowel function as well as reduction in inflammatory markers and increased albumin levels as response to faecal transplantation. In the report of You et al. the patient rapidly displayed normalisation of leucocytosis, stabilisation of blood pressure enabling cessation of vasopressors and improvement in renal function allowing cessation of continuous veno-venous haemofiltration as well as normalisation of bowel function. In the reports of Schwann et al., Gustaffson et al., Persky and Brandt, Aas et al., MacConnachie et al., Khoruts et al., Rholke et al. and Russell et al. cessation of diarrhoea is defined as response. Five of 


\begin{abstract}
these studies also document a change from a positive to a negative Clostridium difficile stool test.
\end{abstract}

For ulcerative colitis, of eight patients reported, all have responded and have remained in remission from 6 months to 13 years $(32,33)$. Patients with ulcerative colitis in the series of Borody et al. responded within one to six weeks and were considered in remission by four months following faecal transplantation (33). Five out of the six patients reported in this series had moderate to severe disease with moderate to severe endoscopic findings. All of the patients were asymptomatic with no endoscopic evidence of active inflammation following faecal transplantation. (Table 3).

\title{
Adverse events
}

No studies of faecal transplantation report any adverse events related to the procedure. Some studies report patient deaths due to the underlying disease where the patient has not responded to the faecal transplantation. In one study in which donor faeces were instilled via a nasogastric tube, the patient died of peritonitis. Although considered unlikely, the nasogastric tube insertion could not be discounted to have been contributory (17). One patient in the study by Silverman et al. developed irritable bowel symptoms following faecal transplantation (25). 


\section{Analysis of effects of faecal transplantation on stool composition and faecal microbiota}

Four studies have attempted to analyse stool before and after faecal transplantation. Using culture, Tvede and Rask-Madsen observed an absence of Bacteroides before bacteriotherapy and during vancomycin therapy whilst patients were symptomatic. During follow up after bacteriotherapy (including faecal enemas in two patients), Bacteroides were regularly cultured (15). Gustafsson et al. studied stool short chain fatty acid concentrations before and after faecal transplantation in nine patients. All short chain fatty acids were found to be reduced in the patient group compared with healthy adults and following faecal enema therapy the relative distribution and absolute amounts of short chain fatty acids returned to patterns similar to healthy adults (18). More recently, using modern molecular 16S rRNA gene sequencing techniques, two studies have shown a significant change in the microbiota following faecal transplantation. Khoruts et al. demonstrated a reduction in Bacteroidetes and Firmicutes in a patient with Clostridium difficile diarrhoea. Following faecal transplantation there was a rapid change in the patient's microbiota to a composition that was highly similar to that of the healthy donor for at least four weeks (the duration of follow-up stool analysis) (28). Grehan et al. undertook analysis on the stool of 10 patients who 
underwent faecal transplantation. A dramatic change was shown in the recipients' microbiota to a composition similar to their donors' microbiota. This study analysed stool from patients up to 24 weeks following faecal transplantation demonstrating a durable change in the recipients' microbiota up to 24 weeks (30).

\section{Conclusions}

Evidence regarding the use of faecal transplantation as a means of modifying the gut microbiota and effecting cure of gastrointestinal illness is accumulating. To date the majority of studies of faecal transplantation have been in fulminant or refractory Clostridium difficile. However, studies of faecal transplantation to date are heterogeneous regarding the patients treated, donors used, optimal screening protocols, methods and frequency of administration, and definition of response.

Furthermore, reports to date may suffer from reporting bias of positive outcomes and under-reporting of adverse effects.

Faecal transplantation, a therapy used for more than half a century, could hold great promise as a future treatment where a dysbiosis of the gut microbiota is responsible for disease. This therapy is inexpensive as well as being effective in some cases. Standardised controlled studies are necessary to ascertain the most effective regimen as well as the most acceptable method of treatment. Two randomised controlled studies of faecal 
transplantation in Clostridium difficile are on-going in North America and Europe and results from these are eagerly awaited as well as a study of faecal transplantation in metabolic syndrome. Studies of faecal transplantation for other gastrointestinal diseases where a dysbiosis of the gut microbiota is evident are necessary. Rigorous screening of potential donors is essential as is the use of partners or close relatives as donors to minimise the potential for transmitting disease. Close monitoring and long term follow up are necessary. Combining clinical studies with molecular analysis of the microbiota and the effects on the immune response may significantly enhance our understanding of the gut microbiota and its relationship with health and disease.

Statement of interests:

Dr A Hart has been a speaker for and is on the advisory board for Abbot, Shire and MSD. 


\section{References}

1. Metchnikoff E. The prolongation of life optimistic studies. The English translation edited by PC Mitchell. 1908. GP Putnam's Sons, New York.

2. Sartor RB. Microbial influences in inflammatory bowel diseases. Gastroenterology 2008; 134:577-594

3. Peterson DA, Frank DN, Pace NR, Gordon JL. Metagenomic approaches for defining the pathogenesis of inflammatory bowel disease. Cell Host and Microbe 2008; 417-427.

4. Vrieze A, Holleman F, Zoetendal EG et al. The environment within: how gut microbiota may influence metabolism and body composition. Diabetologia 2010; 53(4): 606-13.

5. Sekirov I, Shannon I, Russell J et al. Gut microbiota in health and disease. Physiol Rev 2010; 90:859-904.

6. Hart AL, Lammers K, Brigidi $\mathrm{P}$ et al. Modulation of human dendritic cell phenotype and function by probiotic bacteria. Gut 2004;53(11):1602-9.

7. $\mathrm{Ng} \mathrm{SC}$, Plamondon $\mathrm{S}$, Kamm MA et al. Immunosuppressive effects via human intestinal dendritic cells of probiotic bacteria and steroids in the treatment of acute ulcerative colitis. Inflamm Bowel Dis 2010;16(8):1286-98. 
8. Hart AL, Stagg AJ, Kamm MA. Use of probiotics in the treatment of inflammatory bowel disease. J Clin Gastroenterol. 2003 Feb;36(2):111-9.

9. Preidis GA, Versalovic J. Targeting the human microbiome with antibiotics, probiotics and prebiotics: gastroenterology enters the metagenomic era. Gastroenterology 2009; 136:2015-2031.

10. Shannahan F. Probiotics in perspective. Gastroenterology 2010; 139:1808-1812.

11. Rager KD, George LW, House JK, DePeters EJ. Evaluation of rumen transfaunation after surgical correction of left-sided displacement of the abomasum in cows. J Am Vet Med Assoc. 2004; 225(6):915-20

12. Eiseman B, Silen W, Bascom GS et al. Fecal enema as an adjunct in the treatment of pseudomembranous enterocolitis. Surgery 1958; 44:854-859.

13. Bowden TA, Mansberger AR Jr, Lykins LE. Pseudomembranous enterocolitis: mechanism of restoring floral homeostasis. Am Surg 1981; 47: 17883.

14. Schwan A, Sjolin S, Trottestam U. Relapsing Clostridium difficile enterocolitis cured by rectal 
infusion of homologous faeces. The Lancet 1983; 2:845.

15. Tvede M, Rask-Madsen J. Bacteriotherapy for chronic relapsing Clostridium difficile diarrhoea in six patients. The Lancet 1989; 1156-1160.

16. Borody TJ, George L, Andrews P et al. Bowel-flora alteration: a potential cure for inflammatory bowel disease and irritable bowel syndrome? Medical Journal Australia 1989; 150(10): 604.

17. Paterson DL, Iredell J, Whitby M. Putting back the bugs: bacterial treatment relieves chronic diarrhoea. Medical Journal Australia 1994; 160: 232-233.

18. Gustaffson A, Lund-Tonnesen S, Berstad A et al. Faecal short-chain fatty acids in patients with antibioticassociated diarrhoea, before and after faecal enema treatment. Scand J Gastroenterol 1998;33: 721-727.

19. Persky SE and Brandt LJ. Treatment of recurrent Clostridium difficile-associated diarrhoea by administration of donated stool directly through a colonoscope. Am J Gastroenterology 2000; 95: 32833285.

20. Aas J, Gessert E, Bakken JS. Recurrent Clostridium difficile colitis: Case series involving 18 patients treated 
with donor stool administered via a nasogastric tube.

Clinical Infectious Diseases 203; 36: 580-585

21. You DM, Franzos MA. Successful treatment of fulminant Clostridium difficile infection with fecal bacteriotherapy. Annals of Internal Medicine 2008; 148 (8): 632-633.

22. MacConnachie AA, Fox R, Kennedy DR, Seaton RA. Faecal transplant for recurrent Clostridium difficileassociated diarrhoea: a UK case series. Q J Med 2009; 102: 781-784.

23. Rubin TA, Fessert CE, Aas J. Stool transplantation for older patients with Clostridium difficile infection. JAGS 2009; 57 (12) 2386-2387.

24. Russell G, Kaplan J, Ferraro MJ, Michelow IC. Fecal bacteriotherapy for relapsing Clostridium difficile infection in a child: A proposed treatment protocol. Paediatrics 2010; 126(1):e239-e242.

25. Silverman MS, Davis I, Pillai D. Success of selfadministered home fecal transplantation for chronic Clostridium difficile infection. Clinical Gastroenterol Hepatol. 2010; 8: 471-473.

26. Rohlke F, Surawics CM, Stollman N. Fecal flora reconstitution for recurrent Clostridium difficile 
infection: Results and methodology. J Clin

Gastroenterol 2010; 44(8): 567-70

27. Yoon SS, Brandt LJ. Treatment of refractory/recurrent

C. difficile-associated disease by donated stool

transplant via colonoscopy. A case series of 12 patients.

J Clin Gastroenterol 2010; 44(8): 562-66.

28. Khoruts A, Dicksved J, Jansson J, Sadowsky MJ.

Changes in the composition of the human fecal microbiome after bacteriotherapy for recurrent Clostridium difficile-associated diarrhoea. J Clin.

Gastroenterol. 2010; 44(8): 354-360.

29. Garborg K, Waagsbo B, Stalleman A et al. Results of faecal donor instillation therapy for recurrent Clostridium difficile-associated diarrhoea. Scandinavian Journal of Infectious Diseases 2010; 42: 857-861.

30. Grehan MJ, Borody TJ, Leis SM et al. Durable alteration of the colonic microbiota by the administration of donor fecal flora. J Clin Gastroenterol 2010; 44(8): 551-561.

31. Andrews PJ, Barnes P, Borody TJ et al. Chronic constipation reversed by restoration of bowel flora. A case and a hypothesis. Eur J Gastroenterol Hepatol. 1992; 4: 245-247. 
32. Bennet JD, Brinkman M. Treatment of ulcerative colitis by implantation of normal colonic flora. The Lancet 1989; $1: 164$.

33. Borody TJ, Warren EF, Leis $\mathrm{S}$ et al. Treatment of ulcerative colitis using fecal bacteriotherapy. J Clin Gastroenterol 2003; 37(1): 42-47.

34. Bakken JS. Fecal bacteriotherapy for recurrent Clostridium difficile infection. Anaerobe 2009; 15:285289. 
R2

Review article: Faecal transplantation. Tables.

Table 1. Suggested screening investigations

\begin{tabular}{|ll|}
\hline Sample & Investigation \\
Blood & Full Blood Count, Liver Function Tests \\
& Hepatitis A,B,C \\
& HIV 1+2, HTLV I/II \\
& CMV, EBV \\
& Treponema pallidum \\
Stool & \\
& Selective stool culture \\
Clostridium difficile toxin A and B & Microscopy for ova, cysts and parasites \\
\hline
\end{tabular}

Table 2. Methods of faecal transplantation

\begin{tabular}{|l|l|l|}
\hline & Upper Gastrointestinal Tract & Lower Gastrointestinal Tract \\
\hline $\begin{array}{l}\text { Donor stool collection prior to } \\
\text { transplantation }\end{array}$ & $\leq 6$ hours $(13,20,22,24,30)$ & $\leq 24$ hours $(14,17,25-27,30,33)$ \\
\hline Bowel cleansing & No $(13,20,22,24,30)$ & $\begin{array}{l}\text { Yes (colonic instillation) }(26- \\
28,20,32,33)\end{array}$ \\
\hline Donor stool volume & $30-50 \mathrm{~g}(20,22,24,29)$ & $10-200 \mathrm{~g}(15,18,19,21,25-30,33)$ \\
\hline Volume of dilution in saline & $50-250 \mathrm{ml}(20,22,24,29)$ & $20-500 \mathrm{ml}(15,17-19,21,25-30,33)$ \\
\hline Volume instilled & $25-200 \mathrm{ml}(20,22,24,29)$ & $20-500 \mathrm{ml}(15,17-19,21,25-30,33)$ \\
\hline Repeated instillation & No (13,20,22,24) & No $(18,19,26,27,28)$ \\
& Yes $(29)$ & Yes $(12-14,17,25,29,30,33)$ \\
\hline
\end{tabular}


R2

Table 3. Summary of the outcome studies of faecal transplantation

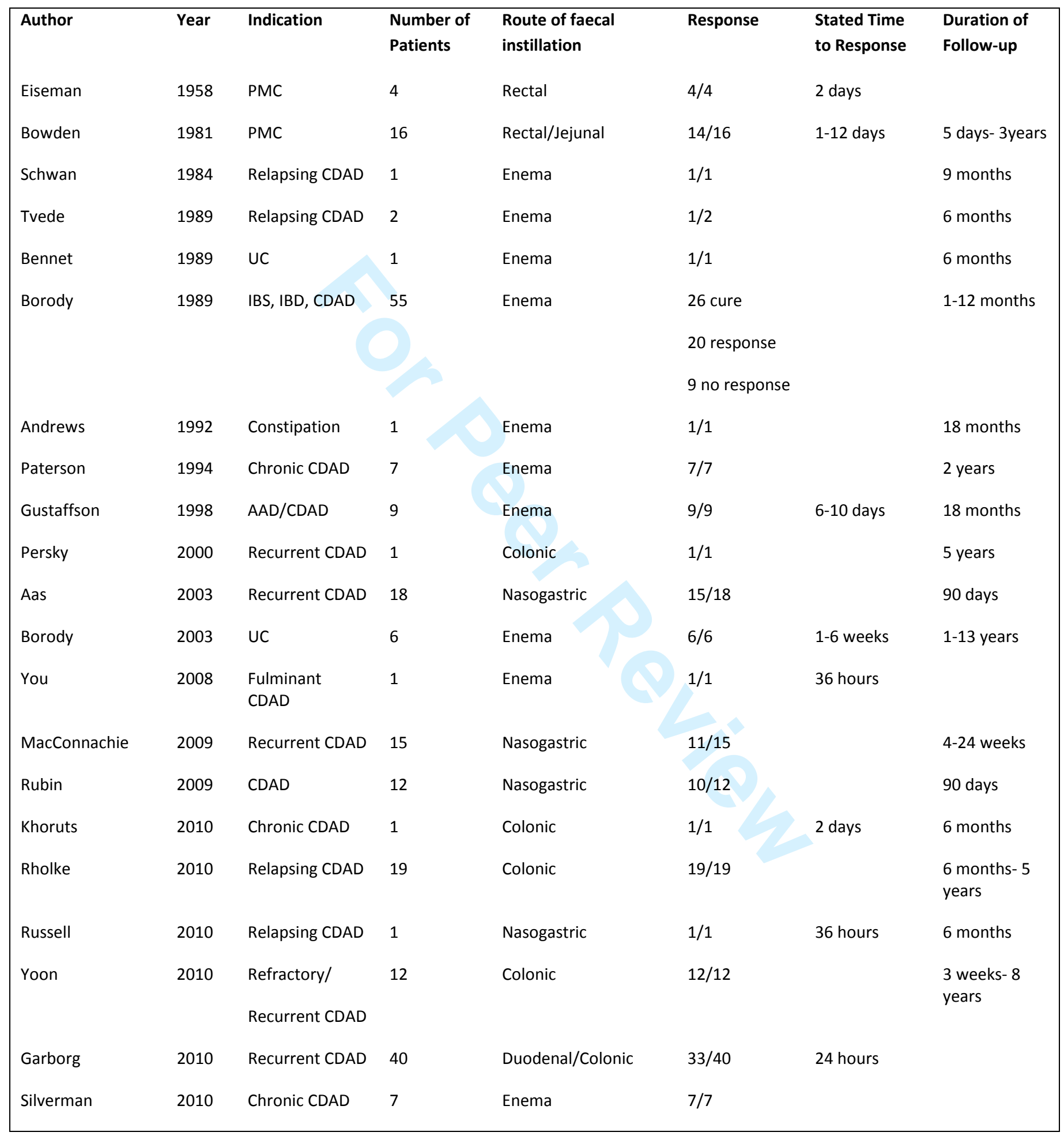


R2

PMC- Pseudomembranous colitis; CDAD- Clostridium difficile associated diarrhoea; AAD- antibiotic associated diarrhoea; IBS- Irritable bowel syndrome; IBD- Inflammatory bowel disease; UC- ulcerative colitis. 